\title{
ARCHIPIÉLAGO DE LOS CHONOS: NUEVOS REGISTROS ARQUEOLÓGICOS Y BIOANTROPOLÓGICOS EN LOS CANALES SEPTENTRIONALES. ISLA TRAIGUÉN, REGIÓN DE AISÉN
}

\author{
OMAR REYES BÁEZ*, MANUEL SAN ROMÁN* Y MAURICIO MORAGA"*
}

\begin{abstract}
RESUMEN
Se dan a conocer nuevos registros de la ocupación humana en los canales septentrionales de Aisén, específicamente en la costa noroccidental de isla Traiguén, en donde se detectaron cuatro sitios arqueológicos. La frecuencia de hallazgos y el notable volumen de uno de los yacimientos registrados sugieren la importancia de dicha área para el asentamiento humano indígena. Los sitios corresponden a corrales de pesca y campamentos caracterizados por importantes acumulaciones de conchal, destacando el hallazgo de osamentas humanas históricas en uno de éstos. Los fechados obtenidos para un sitio de habitación con evidencias de inhumación permiten sostener un uso intensivo de la localidad desde los últimos 1300 años.
\end{abstract}

PALABRAS CLAVE: Patagonia, Archipiélago de los Chonos, isla Traiguén, restos humanos.

\section{CHONOS ARCHIPELAGO: NEW ARCHAEOLOGICAL AND BIOANTHROPOLOGICAL RECORDS IN THE NORTHERN CHANNELS. TRAIGUEN ISLAND, XI REGION DE AISÉN.}

\section{ABSTRACT}

We present new records of human occupation in the northern channels of Aisén, specifically the northwest coast of the Traiguén Island, where four archaeological sites were found. The frequency of the findings and a significant volume of deposits suggest the importance of this area for indigenous human settlement. The sites are fish corrals and camping sites characterized by large accumulations of shell middens, emphasizing the discovery of historical human remains in one of these. The dates obtained for a domestic site with burial remains can sustain the intense use of this locality during the last 1300 years.

KEY WORDS: Patagonia, Chonos Archipelago, Traiguen island, human remains. 


\section{INTRODUCCIÓN}

La investigación arqueológica en la extensa área de los canales septentrionales se ha remitido principalmente a muestreos y prospecciones acotadas en sectores de Chiloé (Legoupil 2005, Rivas y Ocampo 2010), seno de Reloncaví (Gaete et al. 2004), archipiélago de las Guaitecas (Ocampo y Aspillaga 1984, Porter 1993), canal Jacaf y Puyuhuapi (Reyes et al. 2007) y archipiélago Guayaneco (Carabias 2009, Legoupil et al. 2007), lográndose esbozos de un panorama regional que enfatiza la discusión sobre la antigüedad del proceso de colonización y poblamiento litoral sudamericano (Diaz y Garretón 1972-73, Legoupil y Fontugne 1997, Ocampo y Rivas 2004, Orquera y Piana 2006, Rivas et al. 1999) aunque también, en menor medida, se han estudiado temas específicos en torno a subsistencia (Gaete y Navarro 2004, Legoupil 2005, Legoupil et al. 2007, Reyes et al. 2007), características de los grupos cazadores recolectores marinos (Aspillaga et al. 2006), la posibilidad de contactos con grupos de tradición esteparia (Reyes et al. 2009, 2010) y circulación de materias primas (Munita 2007, Stern y Porter 1991, Stern y Curry 1995, Stern et al. 2002). Ciertamente lo fragmentario y disperso de las investigaciones arqueológicas en esta gran área del Pacífico sur, explican esta visión más generalista en cuanto a procesos que respecto a un detallado estudio intrasitio.

En el marco de nuestras investigaciones, y en relación a la búsqueda de registros que permitan explicar los procesos de poblamiento y distribución de grupos humanos en los canales norpatagónicos, es que se torna particularmente sensible la búsqueda, registro y análisis de restos óseos que permitan caracterizar genética, bioantropológica y cronológicamente a estos cazadores recolectores marinos, en especial los denominados históricamente como Chono (Alvarez 2002, Cooper 1946, Martinic 2005). El determinar si es una entidad genéticamente diferenciada de otras poblaciones canoeras de más al sur (Yámana y Kawésqar) o si es producto de una larga historia microevolutiva y/o la expansión tardía de grupos de más al norte (Huilliches desde el área de Chiloé, García et al. 2004), es un tema fundamental respecto a la discusión arqueológica sobre poblamiento, movilidad, cronología y distribución de grupos humanos en el extremo sur de Sudamérica
(Moraga et al. 2000, 2009). Además, la inclusión de análisis bioantropológicos, permitirá incorporar criterios morfológicos, de modos de vida y dietarios (isótopos) que, sin duda, son necesarios no sólo en esta reconstrucción del poblamiento y movilidad en los canales norpatagónicos, sino que también en temáticas específicas como subsistencia y tipo de dieta (p.e. Aspillaga et al. 2006, Barberena 2002, Rodríguez et al. 2007). La búsqueda de depósitos arqueológicos, por otro lado, contribuye a discutir y evaluar culturalmente dichos procesos (dispersión, microevolución) en función de variables como los tipos de ocupación y asentamientos, explotación de recursos, diferencias tecnológicas, circulación de materias primas, contextualización/diversidad de la funebria y en los patrones de distribución espacial y cronológicos de estos contextos inhumatorios (secuencias culturales).

A este respecto, se seleccionó la localidad de isla Traiguén, situada al sur del fiordo Aisén, como punto de partida de la búsqueda de evidencia arqueológica y bioantropológica. En efecto, se encuentran importantes referencias en los diarios del capitán Enrique Simpson (1875) en épocas de reconocimiento geográfico de la República, acerca de la presencia de restos óseos humanos expuestos en perfiles erosionados en los depósitos de conchal al sur de la isla y en los canales aledaños. Las menciones reparan igualmente en que no son pocos los hallazgos bioantropológicos y que su destrucción es significativa por la acción de las mareas -tal como lo comprueba posteriormente Curry (1997)-, y de una tectónica que ha producido hundimiento y solevantamiento de las costas con el consiguiente deterioro de los sitios arqueológicos (Bird 1993, Delgado 2004, Ocampo y Aspillaga, 1984, Porter 1993, Ramos 2005, Reyes et al. 2007, Rivas et al. 1999).

La investigación arqueológica por otro lado, también coincide con estas primeras apreciaciones y hace referencia de conchales con osamentas humanas en la misma área (Curry 1997), además de registrar la procedencia geológica de materias primas sobre artefactos encontrados en el intermareal, estableciendo la proveniencia de algunas de éstas desde la fuente de obsidiana del volcán Chaitén (Stern y Curry 1995). Esta, presenta una amplia circulación en los canales septentrionales (Munita 2007, Reyes et al. 2007, Stern y Porter 1991). Desde su centro en la localidad de Chaitén, es posible encontrarla 
en el área de Chan Chan en la costa de la Décima Región de los Lagos, a más de 400 km de distancia al norte (Stern et al. 2002) hasta la costa sur de isla Traiguén, casi $400 \mathrm{~km}$ más al sur, quedando en evidencia el amplio rango de movilidad y/o intercambio de estos grupos a lo largo del eje costero.

\section{EXPLORACIÓN ARQUEOLÓGICA}

A partir de los antecedentes expuestos, se seleccionó como punto de partida para iniciar el trabajo de exploración arqueológica la costa noroccidental de isla Traiguén, específicamente la localidad conocida como Las Mentas. El área se emplaza hacia la salida sur del fiordo Aisén, entre los canales Errázuriz por el oeste y Costa por el este ( $45^{\circ}$, Fig.1). La isla constituye una de las escasas porciones de tierra emergida (además de isla Nalcayec e itsmo de Ofqui) de la Depresión Central, continuación de la Depresión Intermedia de la zona central del país. Al igual que en la mayor parte del área de los canales septentrionales, se registra un ambiente insular de bosque siempreverde caracterizado por la ecorregión Provincia Templada Húmeda Insular (CfKni) con regímenes de alta pluviometría y oscilaciones térmicas moderadas por la acción del mar (SERPLAC XI Región, 2005).

En el lugar, habita una familia de colonos y pescadores que conforman la comunidad Huilliche "Nahuelquín Delgado" quienes hicieron una denuncia sobre el hallazgo de osamentas en el sector. De acuerdo a los antecedentes expuestos previamente, se decidió realizar una inspección arqueológica a modo de comprobar los datos, hacer un reconocimiento del área y tomar muestras, con el fin de realizar una evaluación previa que permitiera discriminar el potencial arqueológico del sector para planificar nuevas etapas de investigación.

Los trabajos arqueológicos de reconocimiento del área permitieron la identificación y registro de dos conchales arqueológicos además de dos corrales de piedra. A continuación se presentan las principales características de los sitios detectados.

\section{Sitio Nahuelquín 1}

El sitio arqueológico Nahuelquín 1, se encuentra en la costa noroccidental de isla Traiguén $\left(45^{\circ} 27^{\prime} S-73^{\circ} 41^{\prime} \mathrm{W}\right)$. Está formado por un gran depósito de conchal situado en una pequeña bahía reparada de los vientos del oeste. Presenta una costa rocosa hacia su extremo noroeste y una pequeña playa hacia el sector sur. El sector este corresponde al bosque siempre verde escarpado de la isla. El conchal sigue la orientación de la pequeña península que conforma la bahía ${ }^{1}$. Su extensión norte-sur es de $30 \mathrm{~m}$ y un ancho entre $8 \mathrm{~m}$ (sector norte) y 15 $\mathrm{m}$ (sector sur) cubriendo un área aproximada de $345 \mathrm{~m}^{2}$. Se emplaza a $1 \mathrm{msnm}$. No obstante, el sitio se extiende más allá de los límites del conchal, registrándose un instrumento lítico (punta bifacial lanceolada) en el intermareal que se encuentra inmediatamente al sur. Los barrenos practicados en el sitio, arrojaron una profundidad máxima de $4 \mathrm{~m}$ de espesor en la cima, la que va disminuyendo hacia la periferia con profundidades variables. En el borde oeste de este gran conchal, se registró un perfil expuesto en donde afloraban restos óseos humanos, específicamente la bóveda craneana de un individuo que presentaba evidentes huellas de intemperización (cubierto de musgo y blanqueamiento de superficie).

Las actividades en terreno consistieron en la limpieza del perfil y la recuperación de los restos humanos expuestos, además de una intervención con barreno para determinar la potencia estratigráfica del conchal, de manera de registrar toda la matriz y obtener una muestra orgánica datable desde la base que permitiera establecer el momento inicial de la ocupación de la bahía.

La secuencia estratigráfica registrada en este sector del sitio alcanzó los $3 \mathrm{~m}$, describiéndose la siguiente sucesión estratigráfica: Estrato $1(0-40 \mathrm{~cm})$ Densa capa vegetal compuesta por tierra orgánica, café oscura, con pocos clastos pequeños. Estrato 2 (40-50 cm). Interfase entre la capa orgánica y el comienzo del conchal. Estrato $3(50-300 \mathrm{~cm})$ Conchal compuesto principalmente por picorocos (Austromegabalanus psittacus), cholgas (Aulacomya ater) y choros (Mytilus edulis chilensis), además de restos óseos de pescado (vértebras y espinas). La estratigrafía no es homogénea presentando capas muy compactas y duras compuestas por lentes de picorocos, como también sectores en donde las conchas se intercalan con sedimentos orgánicos

1 Sobre este depósito conchal, se instaló la comunidad indígena Nahuelquín Delgado. 


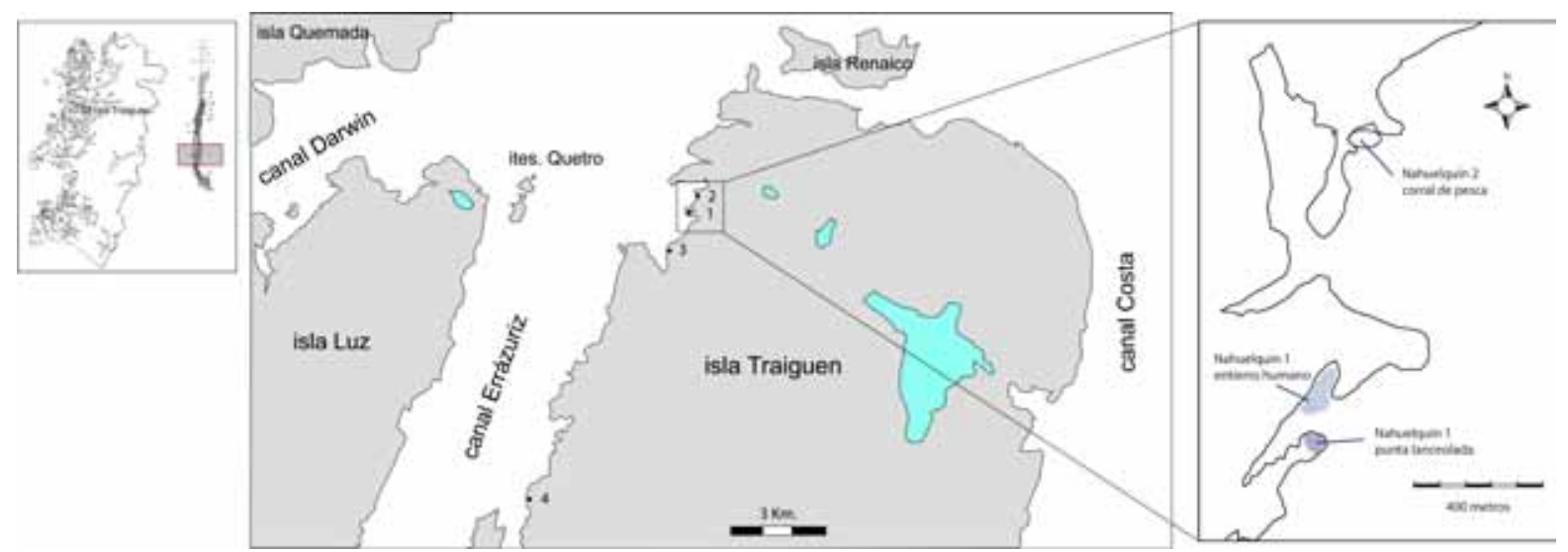

Fig. 1. Contexto de ubicación de los sitios arqueológicos identificados en la costa noroccidental de isla Traiguén, en los canales septentrionales de la Región de Aisén; 1. Nahuelquín 1, 2. Nahuelquín 2, 3. Nahuelquín 3 y 4 . Nahuelquín 4.

(formación de suelos y pisos de ocupación). Hacia la base del depósito, las conchas se encuentran muy molidas y se mezclan con materia orgánica del suelo enterrado bajo el depósito de conchal. Se recuperaron además algunas espículas de carbón en diferentes niveles, sin embargo, no se distinguieron lentes de fogón claramente discernibles en el perfil. Tampoco se observaron materiales culturales durante el tamizado de sedimentos.

Sobre los restos humanos y materiales culturales

La inhumación se encuentra entre los $75 \mathrm{~cm}$ y los $90 \mathrm{~cm}$ desde la superficie actual. Se infiere una posición de entierro orientada norte (pies-pelvis) Sur (cabeza) a partir de la disposición de elementos óseos observables en el perfil. Los restos humanos corresponden a la bóveda craneana, meteorizada y expuesta a la humedad ambiente, por lo que presentan un color blanco y verde (por acción de los musgos). Se registraron además otros elementos anatómicos como un fragmento de escápula y fragmentos de una vértebra cervical y una dorsal. En perfil se observaron más vértebras y la diáfisis de un húmero que quedaron in situ. A juzgar por los elementos anatómicos presentes y por el registro del perfil despejado, se trataría de un entierro primario e individual de un adulto de edad y sexo indeterminados.

Se recuperaron fragmentos de huesos de la escápula para fechar por ${ }^{14} \mathrm{C}$ AMS y realizar análisis de isótopos estables ${ }^{13} \mathrm{C}$ y ${ }^{15} \mathrm{~N}$ que permitan aproximarnos a la dieta del individuo (Ambrose y Katzenberg 2000). También se obtuvo una muestra desde la base del conchal, por medio de barreno, de manera de comenzar a entender una secuencia de ocupación del sector en dos momentos específicos; inicio de depósito conchal y el evento de inhumación.

El registro realizado en la playa y el intermareal situados inmediatamente al sur del extenso conchal (Fig. 2), permitió la recuperación de una punta lanceolada fragmentada elaborada sobre obsidiana riolítica de color gris, materia prima proveniente del volcán Chaitén, situado 400 km más al norte y documentada desde las costas de Chan Chan hasta el sur de isla Traiguén (Stern y Curry 1995, Stern et al. 2002).

Junto con el registro del sitio Nahuelquín 1 se realizó una breve prospección de parte de la costa noroeste de isla Traiguén, de manera de determinar la presencia de otros sitios arqueológicos.

\section{Sitio Nahuelquín 2}

Se trata de un antiguo corral de pesca a $500 \mathrm{~m}$ al norte del sitio Nahuelquín 1 (45²7'S$\left.73^{\circ} 42^{\prime} \mathrm{W}\right)$. Se emplaza en una pequeña bahía de exposición norte, que posee una extensa franja de intermareal. Corresponde a una continuidad de grandes y medianos bloques de piedra sobrepuestos, obtenidos del entorno. Corren en dirección suroeste-noreste en forma elíptica por cerca de 25 m. Parte de esta acumulación antrópica de piedras se encuentra a más de 2 msnm actual, habiendo 


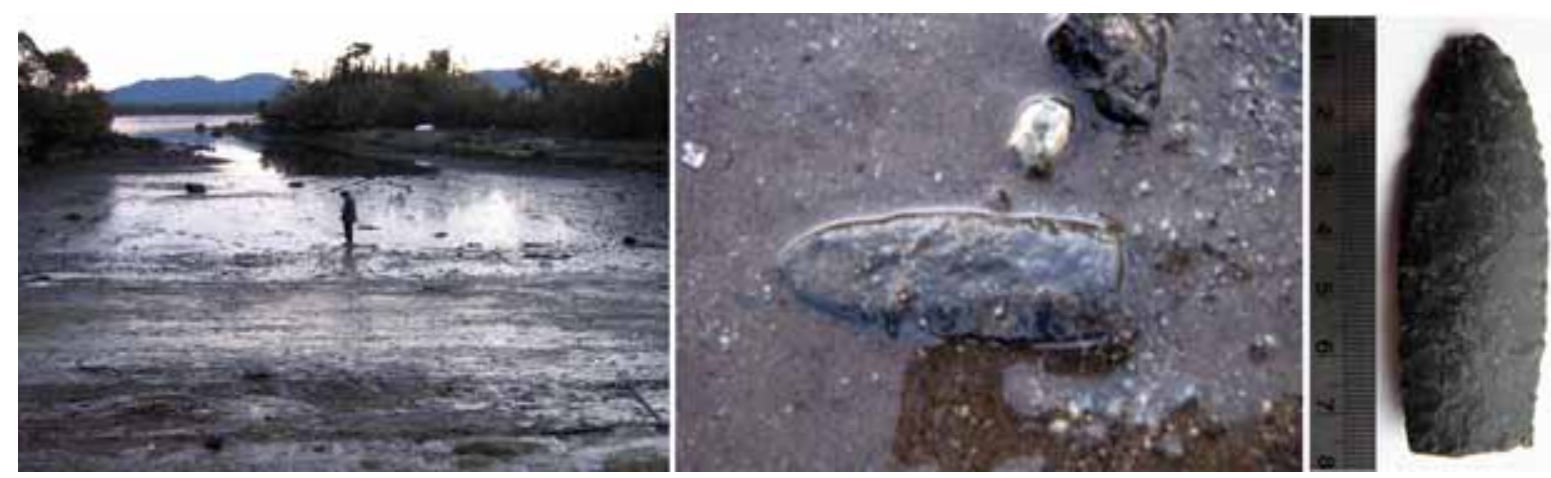

Fig. 2. (Izq.) Posicionamiento del punto de descubrimiento de la punta lanceolada en el intermareal situado inmediatamente al sur del conchal, sitio Nahuelquín 1. (Centro). Vista del cabezal lítico in situ. (Der.) Vista de la morfología del instrumento elaborada sobre obsidiana riolítica proveniente del volcán Chaitén.

sido colonizadas por líquenes y juncos. Bien puede deberse a solevantamiento del terreno producidos por cambios locales de acuerdo a condiciones tectónicas, sea de hundimiento o levantamiento, ya registradas para otros sitios en la Región (Bird 1993, Carabias 2009, Legoupil 2005, Porter 1993, Ramos 2005, Reyes et al. 2007). La información del colono Benjamín Nahuelquín es que, al menos hace 50 años, ya se encontraban los corrales en esa misma condición.

\section{Sitio Nahuelquín 3}

También corresponde a un antiguo corral ubicado $700 \mathrm{~m}$ al sur del sitio Nahuelquín 1 (45⒉ $28^{\prime} \mathrm{S}$ $\left.73^{\circ} 41^{\prime} \mathrm{W}\right)$. Se emplaza en un sector donde la costa conforma una bahía muy estrecha, a manera de poza, con exposición noroeste. Al igual que la descripción del corral anterior, se trata de una continuidad de grandes bloques pétreos que corren, en forma elíptica, en un eje suroeste-noreste a lo largo de $70 \mathrm{~m}$, sobre $1 \mathrm{~m} . s . n . m$. También se observan

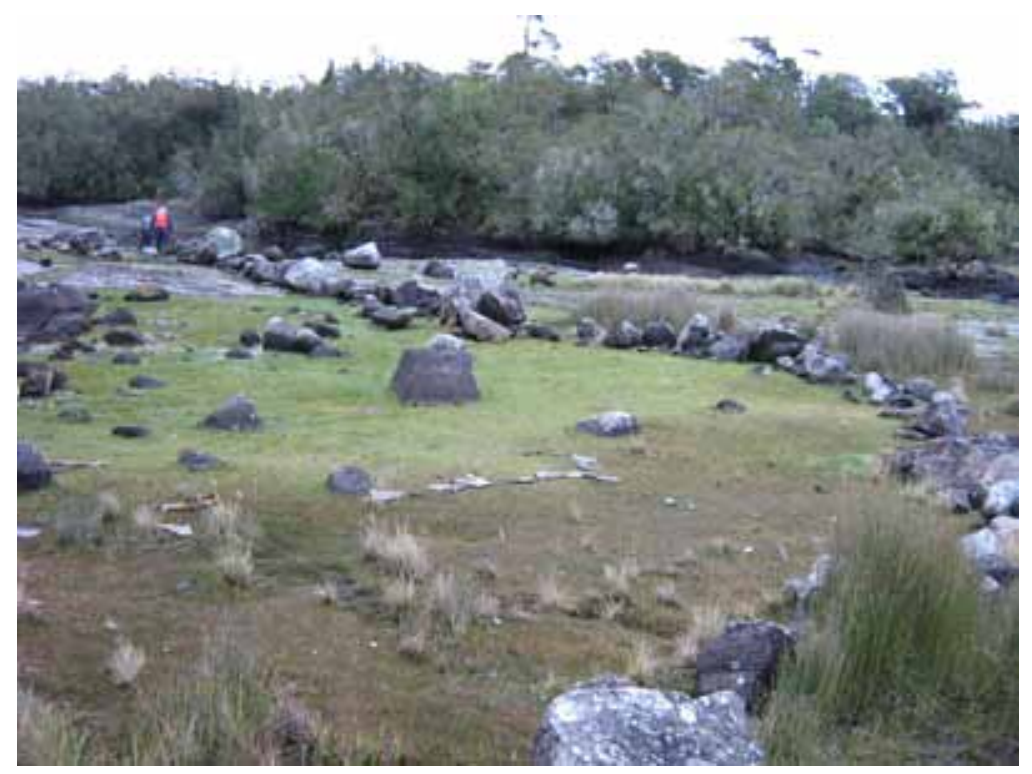

Fig. 3. Sitio Nahuelquín 3. Nótese como la hilera de rocas que conforman este corral de pesca, se encuentra sobre la línea de alta marea. Las rocas han sido colonizadas por líquenes mientras que el área circundante se ha cubierto por pasto y matorrales, atestiguando los cambios morfológicos producidos en la línea costera de ésta área archipelágica. 
los cambios indirectos en los niveles marinos que afectan la costa, por medio de la proliferación de líquenes en las rocas y el pasto que ha crecido junto a éstas (Fig. 3).

Sitio Nahuelquín 4.

Se trata de un pequeño conchal ubicado a unos $8 \mathrm{~km}$ al sur del sector Las Mentas (45 $31^{\circ} \mathrm{S}$ $73^{\circ} 44^{\prime} \mathrm{W}$ ), sobre una pequeña bahía con exposición sur. Las pruebas de barreno evidenciaron que bajo los $5 \mathrm{~cm}$ de la capa vegetal superficial se encuentra una matriz descompuesta de conchal antrópico de 30 a $50 \mathrm{~cm}$ de profundidad, principalmente de valvas de choros (Mytilus edulis chilensis). No se detectaron en los barrenados materiales culturales. La dispersión del conchal sigue la misma dirección que la orientación de la playa sobre la cual se ha depositado, es decir, 30 m en dirección este-oeste, $10 \mathrm{~m}$ en dirección norte-sur y a $5 \mathrm{msnm}$.

\section{RESULTADOS: OCUPACIONES HUMANAS, ISOTOPOS Y CRONOLOGÍA}

La evaluación arqueológica de la costa noroccidental de isla Traiguén no hizo más que corroborar los antecedentes históricos (Simpson 1875) y arqueológicos previos de más al sur (Curry 1997), en cuanto al potencial y diversidad del registro cultural, y su aporte a la discusión del poblamiento costero, movilidad y dispersión de las ocupaciones humanas.

Respecto al sitio Nahuelquín 1 , un extenso y potente depósito de conchal con al menos una inhumación identificada, se obtuvo una muestra de la base del conchal a los $3 \mathrm{~m}$ de profundidad en el sector del entierro, además de otra proveniente de un fragmento de escápula de la inhumación ubicada entre los 75 y $90 \mathrm{~cm}$ (Tabla 1).

La inhumación en el conchal ha sido fechada en $430 \pm 25$ años AP, hasta ahora corresponde al único esqueleto fechado directamente en los canales septentrionales en el archipiélago de los Chonos. No obstante, cabe guardar las distancias respecto a un mayor detalle del tipo de entierro y a las comparaciones con otro tipo de inhumaciones hasta no haber excavado y registrado completamente este hallazgo.

En relación a la señal isotópica de los restos óseos inhumados en isla Traiguén (Tabla 1), y de acuerdo a datos y comparaciones controladas de niveles isotópicos en individuos arqueológicos y en recursos de Pampa y Patagonia (Barberena 2002, 2008, Berón et al. 2009, Borrero et al. 2001, 2009, Tessone et al. 2009), podemos inferir una dieta mixta con aporte de recursos terrestres y marinos. Este resultado es comparable con los valores de isótopos obtenidos para restos humanos, también de postcontacto, provenientes de más al norte, del sitio Puqueldon I (Sáez 2008), situado en isla Lemuy en el archipiélago de Chiloé. Se obtuvieron muestras y se fecharon por ${ }^{14} \mathrm{C}$ AMS dos individuos dando una cronología de $210 \pm 25$ (UGAMS 8291) y $210 \pm 30$ años AP (UGAMS 8292), mientras que los valores de isótopos obtenidos para estos restos son $-20,1 \delta^{13} \mathrm{Ccol} / 8,8 \delta^{15} \mathrm{~N}$ y $-19,7 \delta^{13} \mathrm{Ccol} / 9,3$ $\delta^{15} \mathrm{~N}$, respectivamente. Estos valores, probablemente están reflejando cambios culturales importantes (p.e. movilidad, alimentación) en grupos humanos con tradición insular. Claramente, y no contando en la actualidad con una base de datos regional de los valores isotópicos de los recursos específicos consumidos por los cazadores recolectores marinos en los canales septentrionales, esta información deberá ser cotejada con una adecuada excavación y registro del rasgo fúnebre, además del análisis bioantropológico. Por otro lado, los resultados preliminares de ADN mitocondrial efectuados sobre muestras del individuo de isla Traiguén, permiten adscribirlo al infrecuente sub-haplogrupo D4h3a, detectado en individuos kawésqar actuales (Moraga et al. 2010) y en pobladores de la isla Laitec, en el archipiélago de Chiloé (Moraga et al. 2000). Hasta aquí, la evidencia isotópica resultaría incompatible con los valores esperables para un individuo caza-

Tabla 1. Resultados de las muestras obtenidas.

\begin{tabular}{|c|c|c|c|c|c|}
\hline Código CAIS & Muestra & Material y profundidad & Fechado AP & $\delta^{13} \mathrm{C}(\% 0) \mathrm{col}$ & $\delta^{15} \mathrm{~N}(\% 0)$ \\
\hline UGAMS 04949 & NAH 1-1 & Escápula humana $(75-90 \mathrm{~cm})$ & $430 \pm 25$ & -21.0 & 11.3 \\
\hline UGAMS 04950 & NAH 1-2 & Conchas $(300 \mathrm{~cm})^{2}$ & $1820 \pm 25$ & -1.2 & \\
\hline
\end{tabular}

2 La muestra obtenida de la base del conchal corresponde a pequeños fragmentos de Mytilidae (choros y cholgas) y Balanidae (picoroco). 
dor-recolector marino, aun cuando la información genética sugiere relación con poblaciones insulares de Chiloé y/o kawésqar.

Por otro lado, en relación a la antigüedad del conchal, debemos señalar que la fecha sobre conchas de la base del depósito (1820 25 AP) estaría sujeta a efecto reservorio, pudiendo descontar en aproximadamente 500 años la antigüedad del sitio si utilizamos los valores estimados de dicho efecto para otras latitudes (San Román et al. 2009). De esta manera, la base de la ocupación se remontaría en alrededor de 1300 años AP.

Se esperaba que el inicio de la ocupación de la bahía fuera cercana a 5.000 - 4.000 años AP, ya que la tipología lanceolada del cabezal lítico recuperado en el intermareal (Fig. 2), de amplia distribución en los canales patagónicos (Díaz y Garretón 1972/3, Legoupil 2003, Gaete y Navarro 2004, Morello et al. 2002, Ocampo y Rivas 2004, Orquera et al. 1977, Piana y Orquera 2007, Porter 1993), se asocia a este rango cronológico. Además, la materia prima usada, proveniente de casi $400 \mathrm{~km}$ de distancia desde la fuente del volcán Chaitén, refleja un amplio circuito de movilidad $y / o$ intercambio desde el norte, proceso del cual no hay cronología establecida. No obstante, el que este instrumento haya sido recuperado del intermareal, ejemplifica la posibilidad cierta de ocupaciones más antiguas que han sido "lavadas" por hundimiento $\mathrm{y} / \mathrm{o}$ solevantadas por cambios de la línea costera por una tectónica aún poco documentada (cfr. Legoupil 2005, Porter 1993, Reyes et al. 2007). Ciertamente, esta situación presenta un desafío en cuanto a la búsqueda y resolución de estas ocupaciones, en dónde pueden ser ubicadas y bajo cuales condiciones de emplazamiento, evaluando paralelamente las condiciones geomorfológicas de extensas áreas litorales.

Finalmente, la información obtenida a partir de este trabajo permite plantear nuevos problemas asociados a la ocupación humana del archipiélago septentrional como la anomalía isotópica v/s movilidad histórica desde Chiloé y/o sur de Península de Taitao. Esperamos que el desarrollo de nuevas investigaciones en el área permita avanzar en la resolución de éste y otros temas básicos referidos a la ocupación humana prehistórica y las trayectorias de ésta a lo largo del Holoceno en esta relevante porción de la costa meridional sudamericana.

\section{AGRADECIMIENTOS}

Agradecemos especialmente a la Comunidad Indígena Huilliche Nahuelquín Delgado conformada por Don Benjamín Nahuelquín Levipani, su señora Isabel Delgado y sus hijos (Esau, Eduardo, Manuel, Mónica y Marcia entre otros miembros familiares) por su generosidad, hospitalidad y por las gestiones realizadas para que pudiésemos realizar nuestro trabajo. También nuestro reconocimiento a Leonardo Zuñiga (SERNAGEOMIN XI Región) por su apoyo y gestión. A Patricia Curry por facilitarnos información de sus prospecciones. Financiamiento FONDECYT N 1100643 y 1085329.

\section{BIBLIOGRAFÍA}

ALVAREZ, R. 2002. Reflexiones en torno a las identidades de las poblaciones canoeras, situadas entre los $44^{\circ} \mathrm{y}$ $48^{\circ}$ de latitud sur, denominadas "Chonos". Anales del Instituto de la Patagonia Serie Ciencias Humanas, 30:79-86

AMBROSE, S. y M. KATZENBERG 2000. Biogeochemical Approaches to Paleodietary Analysis. En Advances in archaeological and museum science. Vol. 5. Kluwer Academic Publishers, New York.

ASPILLAGA, E., M. CASTRO, M. RODRIGUEZ y C. OCAMPO 2006. Paleopatología y estilo de vida: El ejemplo de los Chonos. Magallania, 34(1):77-85.

BARBERENA, R. 2002. Los límites del mar. Isótopos estables en Patagonia meridional. Sociedad Argentina de Antropología, Buenos Aires.

2008. Arqueología y biogeografía humana en Patagonia Meridional. Sociedad Argentina de Antropología, Buenos Aires.

BORRERO, L., R. GUICHÓN, R. TYKOT, J. KELLY, A. PRIETO y P. CÁRDENAS 2001. Dieta a partir de isotopos estables en restos óseos humanos de Patagonia austral. Estado actual y perspectivas. Anales del Instituto de la Patagonia, Serie Ciencias Humanas, 29:119-127.

BORRERO, L., R. BARBERENA, N. FRANCO, J. CHARLIN y R. TYKOT 2009. Isotopes and Rocks: Geographical Organisation of Southern Patagonian Hunter-Gatherers. International Journal of Osteoarchaeology, 19:309-327.

BERÓN, M., L. LUNA y R. BARBERENA 2009. Isotopic archaeology in the Western Pampas (Argentina): Preliminary results and perspectives. International Journal of Osteoarchaeology, 19:250-265. 
BIRD, J. 1993. Viajes y arqueología en Chile Austral. Editado por Hyslop J. Ediciones de la Universidad de Magallanes, Punta Arenas.

CARABIAS, D. 2009. Naufragio de la H.M.S. Wager en la Patagonia. Encuentro de dos mundos. Consejo Nacional de la Cultura y las Artes, FONDART, Santiago.

COOPER, J.M. 1946. The Chono. Handbook of South American Indians. En Bureau of American Ethnology Bulletin 143. Vol. 1. Editado por J. Steward. Smithsonian Institution, Washington.

CURRY, P. 1997. Archaeological exploration of the Central Chonos Archipelago. Informe Arqueológico Consejo Monumentos Nacionales, Santiago. MS.

DÍAZ, C.D. y M.C. GARRETÓN 1972/73. El poblamiento prehispánico del área insular septentrional chilena. En: Actas del VI Congreso de Arqueología chilena, pp. 559-584.

DELGADO, S. 2004. Relación entre el perfil del basamento en fiordos y canales y la morfoestructura regional en norpatagonia. Tesis de Magíster en Geografía con mención en Evaluación Territorial. Escuela de Geografía. Facultad de Arquitectura y Urbanismo. Universidad de Chile, Santiago. MS.

GAETE, N. y X. NAVARRO 2004 Estrategias de vida de canoeros cazadores pescadores recolectores del seno de Reloncaví. Entre el bosque siempreverde y el mar interior. Región de Los Lagos, Chile. En: Contra viento y marea. Arqueología de Patagonia, pp. 217-235. Instituto Nacional de Antropología y Pensamiento Latinoamericano y Sociedad Argentina de Antropología, Buenos Aires.

GAETE, N., X. NAVARRO, F. CONSTANTINESCU, R. MERA, D. SELLES, M. SOLARI, L. VARGAS, D. OLIVA y L. DURAN 2004 Una mirada al modo de vida canoero del mar interior desde Piedra Azul. Chungara Revista de Antropología Chilena volumen especial, Tomo I: 333-346.

GARCÍA, F., M. MORAGA, S. VERA, H. HENRÍQUEZ, E. LLOP, C. OCAMPO, E. ASPILLAGA y F. ROTHAMMER 2004. Origen y microdiferenciación de la población humana del Archipiélago de Chiloé. Revista Chilena de Historia Natural, 77: 539-546.

LEGOUPIL, D. 2003. Cazadores-recolectores de Ponsonby (Patagonia austral) y su paleoambiente desde VI al III milenio A. C. Magallania, 31: 63-119, tirada especial.

LEGOUPIL, D. 2005. Recolectores de mariscos tempranos en el sureste de la isla de Chiloé. Magallania, 33(1):51-61.

LEGOUPIL, D., P. BEAREZ, S. LEPETZ, M. SAN ROMÁN y K. SALAS 2007. De la pesca a la caza: Evolución económica del sitio Stuven 1, al sur del Golfo de Pe- nas. Arqueología de Fuego-Patagonia. pp. 279 - 294. Ediciones CEQUA. Punta Arenas, Chile.

LEGOUPIL, D. y M. FONTUGNE 1997. El poblamiento marítimo en los archipiélagos de Patagonia: núcleos antiguos y dispersión reciente. Anales del Instituto de la Patagonia, Serie Ciencias Humanas, 25:75-87.

MARTINIC, M. 2005 De la Trapananda al Áysen. Pehuén Editores, Santiago.

MORAGA, M., P. ROCCO, J. MIQUEL, F. NERVI, E. LLOP, R. CHAKRABORTY, F. ROTHHAMMER y P. CARVALLO 2000. Mitochondrial DNA polymorphisms in chilean aboriginal populations: implications for the peopling of the southern cone of the continent. American Journal of Physical Anthropology, 113:19-29.

MORAGA, M., E. ASPILLAGA y F. MENA 2009. Search for founder mitochondrial lineages in Holocene human remains in Patagonia. American Journal of Physical Anthropology, 48:193-193.

MORAGA M, M. DE SAINT PIERRE, F. TORRES y J. RÍOS 2010. Vínculos de parentesco por vía materna entre los últimos descendientes de la etnia Kawésqar y algunos entierros en los canales patagónicos: evidencia desde el estudio de linajes mitcondriales. Magallania, 38(2):103-114.

MORELLO, F.; M. SAN ROMÁN y A. PRIETO 2002. Puntas de proyectil lanceoladas en Patagonia meridional y Tierra del Fuego. Anales Instituto Patagonia, Serie Ciencias Humanas, 30:155-166.

MUNITA, D. 2007. Materias primas líticas en sitios costeros del extremo sur septentrional de Chile. Dispersión y aprovisionamiento. Arqueología de Fuego-Patagonia. pp. 189-204. Ediciones CEQUA. Punta Arenas, Chile.

OCAMPO, C. y E. ASPILLAGA 1984. Breves notas sobre una prospección arqueológica en los archipiélagos de las Guaitecas y de los Chonos. Revista Chilena de Antropología, 4:155.

OCAMPO, C. y P. RIVAS 2004. Poblamiento temprano de los extremos geográficos de los canales patagónicos: Chiloé e isla Navarino 1. Chungara. Revista de Antropología Chilena Volumen Especial, tomo 1: 317-331.

ORQUERA L.A., A. SALA, E.L. PIANA y A. TAPIA 1977. Lancha Packewaia. Editorial Huemul, Buenos Aires.

ORQUERA L. A. y E.L. PIANA 2006 El poblamiento inicial del área litoral sudamericana sudoccidental. Magallania, 34(2):21-36.

PIANA, E. y L. ORQUERA 2007. Diferencias regionales y temporales en el litoral sudoccidental de Sudamérica. Arqueología de Fuego-Patagonia. pp. 311 - 326. Ediciones CEQUA. Punta Arenas, Chile. 
PORTER, C. 1993. GUA-010, un sitio costero erosionado en una zona sísmica activa. En: Actas del XII Congreso Nacional de Arqueología Chilena, pp. 81-88. Boletín del Museo Regional de la Araucanía 4.

RAMOS, V. 2005 Seismic ridge subduction and topography: Foreland deformation in the Patagonian Andes. Tectonophysics 399: 73-86.

REYES, O., C. MÉNDEZ, A. MALDONADO, H. VELÁSQUEZ, V. TREJO, M. CÁRDENAS y A. ABARZÚA 2009. Uso del espacio de cazadores recolectores y paleoambiente Holoceno en el valle del río Cisnes, Región de Aisén, Chile. Magallania, 37(2):91-107.

REYES, O., C. MÉNDEZ y H. VELÁSQUEZ 2010. De las estepas a los canales septentrionales: uso humano de distintos ambientes/bosques del valle del río Cisnes (Holoceno tardío, XI Región de Aisén). En: Actas del XVII Congreso Nacional de Arqueología Chilena. Tomo 2: 1487- 1495. Valdivia, Chile.

REYES, O., C. MÉNDEZ, M. SAN ROMÁN, P. CÁRDENAS, H. VELÁSQUEZ2, V. TREJO, F. MORELLO y C. STERN 2007. Seno Gala 1; nuevos resultados en la arqueología de los canales septentrionales ( 44ํㅗㅇ XI Región de Aisén, Chile). Magallania, 35(2):91-106.

RIVAS P., C. OCAMPO y E. ASPILLAGA 1999. Poblamiento Temprano de los Canales Patagónicos: El Núcleo Ecotonal Septentrional. Anales del Instituto de la Patagonia. Serie Ciencias. Humanas, 27:221-230.

RIVAS, P. y C. OCAMPO 2010. La adaptación humana al bosque en la isla de Chiloé. Estrategias adaptativas en el litoral septentrional de los canales patagónicos. En: Actas del XVII Congreso Nacional de Arqueología Chilena. Tomo 2: 1449 - 1460. Valdivia, Chile.

RODRÍGUEZ, M., E. ASPILLAGA y B. ARENSBURG 2007. El estudio bioantropológico de las colecciones esqueletales del Archipiélago de Chiloé: Perspectivas y limitaciones. Arqueología de Fuego-Patagonia. pp. 269-278. Ediciones CEQUA. Punta Arenas, Chile.
SÁEZ, A. 2008. Impacto del contacto hispano-indigena en la salud de la población de Chiloé. Un caso de tuberculosis en el cementerio Puqueldon 1. Magallania, 36(2):167-174.

SAN ROMÁN, M; K. SALAS y M. FONTUGNE 2009. Primeros avances en la reconstrucción de secuencias de ocupación de cazadores recolectores marinos en el Estrecho de Magallanes, Patagonia meridional. En Arqueología de la Patagonia Una mirada desde el último confín. Tomo I: 35-45. Editorial Utopías, Ushuaia.

SERPLAC XI Región 2005 Atlas de la Región de Aysén. LOM Ediciones, Santiago.

SIMPSON, E. 1875. Esploraciones hechas por la Corbeta Chacabuco en 1870. Anuario Hidrográfico de la Marina. Tomo I.

STERN, C. y C. PORTER 1991. Obsidiana en yacimientos arqueológicos de Chiloé y las islas Guaitecas. Anales del Instituto de la Patagonia. Serie Ciencias Humanas, 20:205-209.

STERN, C. y P. CURRY 1995. Obsidiana del sitio Posa Las

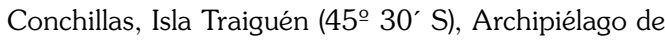
los Chonos. Anales del Instituto de la Patagonia Serie Ciencias Humanas, 23:119-124.

STERN, C., X. NAVARRO y J. MUÑOZ 2002. Obsidiana gris translúcida del volcán Chaitén en los sitios arqueológicos de Quilo (Isla Grande de Chiloé) y Chanchán (X Región) Chile, y obsidiana del Mioceno en Chiloé. Anales del Instituto de la Patagonia, Serie Ciencias Humanas, 30: 167-174.

TESSONE, A. F. ZANGRANDO, G. BARRIENTOS, R. GOÑI, H. PANARELLO AND M. CAGNONI 2009. Stable Isotope Studies in the Salitroso Lake Basin (Southern Patagonia, Argentina): Assessing Diet of Late Holocene Hunter-Gatherers. International Journal of Osteoarchaeology, 19:297-308. 
